PROCEEDINGS OF THE

AMERICAN MATHEMATICAL SOCIETY

Volume 127, Number 8, Pages 2325-2333

S 0002-9939(99)04793-0

Article electronically published on April 9, 1999

\title{
FINITE-DIMENSIONAL LEFT IDEALS IN SOME ALGEBRAS ASSOCIATED WITH A LOCALLY COMPACT GROUP
}

\author{
M. FILALI
}

(Communicated by Palle E. T. Jorgensen)

\begin{abstract}
Let $G$ be a locally compact group, let $L^{1}(G)$ be its group algebra, let $M(G)$ be its usual measure algebra, let $L^{1}(G)^{* *}$ be the second dual of $L^{1}(G)$ with an Arens product, and let $L U C(G)^{*}$ be the conjugate of the space $L U C(G)$ of bounded, left uniformly continuous, complex-valued functions on $G$ with an Arens-type product. We find all the finite-dimensional left ideals of these algebras. We deduce that such ideals exist in $L^{1}(G)$ and $M(G)$ if and only if $G$ is compact, and in $L^{1}(G)^{* *}$ (except those generated by right annihilators of $L^{1}(G)^{* *}$ ) and $L U C(G)^{*}$ if and only if $G$ is amenable.
\end{abstract}

\section{InTRODUCTION}

Let $G$ be a locally compact group, $L^{1}(G)$ be its group algebra, and $M(G)$ be its usual measure algebra. Other Banach algebras (usually larger than $L^{1}(G)$ and $M(G)$ ) can also be associated with $G$. For instance, the second dual $L^{1}(G)^{* *}$ of $L^{1}(G)$ is a Banach algebra with an Arens product. One can also consider the space $L U C(G)$ of the bounded left uniformly continuous functions on $G$, or the space $W A P(G)$ of the weakly almost periodic functions on $G$. An Arens-type product can be introduced into the conjugate of each of these spaces of functions and make them into Banach algebras. Let $\mathcal{A}$ denote any of these algebras. Our main concern in this paper is with the finite-dimensional left ideals in $\mathcal{A}$. We start in Theorem 1 by giving examples of such ideals. These examples are obtained with the help of the $U$-invariant elements of $\mathcal{A}^{n}$ where $U$ is a continuous and bounded representation of $G$ on $\mathbb{C}^{n}$. This notion has already been introduced in earlier papers with $n=1$. In [3], the so-called $\chi$-invariant elements (where $\chi$ is a character of $G$ ) were introduced in order to determine the minimal left ideals of these algebras when $G$ is abelian. In [4], these type of elements were referred to as $\lambda$-invariant elements with $\lambda \in \mathbb{T}$, and were used to solve some linear equations in $\ell^{\infty}(\mathbb{Z})^{*}$, then to determine the finite-dimensional left ideals in this case. In [5], the $U$-invariance was used to study the minimal ideals in these algebras. In Theorems 2 and 3 , we determine all the finite-dimensional left ideals in $\mathcal{A}$ and show that they are in general of the form given in Theorem 1. This is a generalization of the result obtained in [4, 2.7(a)]. We deduce in the corollary which follows that such ideals exist in $\operatorname{LUC}(G)^{*}$ and in $L^{1}(G)^{* *}$ (apart from those generated by right annihilators of $L^{1}(G)^{* *}$ ) if and only

Received by the editors October 28, 1997.

1991 Mathematics Subject Classification. Primary 43A10, 22D15.

Key words and phrases. Locally compact group, Arens product, representation, amenable, $U$-invariant, finite-dimensional left ideal. 
if $G$ is amenable. In $L^{1}(G)$ and $M(G)$, the ideals of finite-dimension exist if and only if $G$ is compact.

\section{Preliminaries}

Let $G$ be a locally compact group with a left Haar measure $\lambda$, and a Haar modular function $\Delta$. For measurable functions $f$ and $\phi$ on $G$ and for $s \in G$, we write

$$
f * \phi(s)=\int_{G} f(t) \phi\left(t^{-1} s\right) d \lambda(t)
$$

whenever the integral exists. We shall be concerned with the following Banach algebras which are related to $G$. We begin by recalling, of course, the most intimate ones: the group algebra $L^{1}(G)$ and the measure algebra $M(G)$. The first one is the Banach algebra of all measurable complex-valued functions $\phi$ on $G$ satisfying

$$
\int_{G}|\phi(s)| d \lambda(s)<\infty
$$

The product of two elements $\phi$ and $\psi$ in $L^{1}(G)$ is $\phi * \psi$. The second one is the Banach algebra of all bounded, regular, Borel measures on $G$. By the Riesz representation theorem (see [8, Chapter 3]), we shall regard a measure in $M(G)$ as an element of $C_{0}(G)^{*}$, where $C_{0}(G)$ is the space of continuous complex-valued functions on $G$ vanishing at infinity. The product of two elements $\mu$ and $\nu$ of $M(G)$ is given then by

$$
(\mu \nu)(f)=\int_{G} \int_{G} f(s t) d \mu(s) d \nu(t)=\int_{G} \int_{G} f(s t) d \nu(t) d \mu(s) \quad \text { for } \quad f \in C_{0}(G) .
$$

Furthermore, for each $\phi \in L^{1}(G)$, we define $\lambda_{\phi} \in M(G)$ by

$$
\lambda_{\phi}(f)=\int_{G} f(s) \phi(s) d \lambda(s) \quad \text { for } \quad f \in C_{0}(G) .
$$

Then under the map $\phi \rightarrow \lambda_{\phi}$, we will regard $L^{1}(G)$ as a subalgebra of $M(G)$. In fact, $L^{1}(G)$ is a closed two-sided ideal of $M(G)$ (see for example [8, Theorem 19.18]). The other algebras which we shall consider are defined in the following way. Let $L^{\infty}(G)$ be the Banach space of all measurable complex-valued functions that are bounded almost everywhere with respect to $\lambda$, and for each $\phi \in L^{\infty}(G)$, let $\hat{\phi}$ be the function defined on $G$ by $\hat{\phi}(s)=\Delta\left(s^{-1}\right) \phi\left(s^{-1}\right)$. The Banach space $L^{1}(G)^{* *}$ becomes a Banach algebra with the first Arens product. This product is obtained by first letting

$$
f_{\nu}(\phi)=\nu(\hat{\phi} * f) \quad \text { for all } \quad \nu \in L^{1}(G)^{* *}, f \in L^{\infty}(G) \text { and } \phi \in L^{1}(G) .
$$

Then, for $\mu$ and $\nu$ in $L^{1}(G)^{* *}$,

$$
(\mu \nu)(f)=\mu\left(f_{\nu}\right) \text { for all } f \in L^{\infty}(G) .
$$

Let $C(G)$ denote the space of all bounded, complex-valued, continuous functions on $G$. The left translate of a function $f$ on $G$ by $s \in G$ is defined by $f_{s}(t)=f(s t)$ for all $t \in G$. Let $\operatorname{LUC}(G)$ be the space of left uniformly continuous functions in $C(G)$, i.e.,

$$
L U C(G)=\left\{f \in C(G): s \mapsto f_{s}: G \rightarrow C(G) \text { is norm continuous }\right\} .
$$


Then $L U C(G)^{*}$ is also a Banach algebra under the product

$$
\begin{aligned}
(\mu \nu)(f) & =\mu\left(f_{\nu}\right) \quad \text { for all } \quad f \in L U C(G), \quad \text { where } \\
f_{\nu}(s) & =\nu\left(f_{s}\right) \quad \text { for all } \quad s \in G
\end{aligned}
$$

(the function $f_{\nu}$ is easily seen to be in $L U C(G)$ ). Note that the product in $M(G)$ (and so in $L^{1}(G)$ ) is defined in the same way with $C_{0}(G)$ instead of $L U C(G)$.

More generally, one can start with a norm closed, conjugate closed subspace $F$ of $C(G)$ containing the constant functions and having the property that the functions $f_{s}$ and $f_{\mu}$ are in $F$ for all $f \in F, s \in G$ and $\mu \in F^{*}$ (the functions $f_{s}$ and $f_{\mu}$ are defined as earlier in $L U C(G)$ ). Following [2, Definition 2.2.10], such an $F$ is said to be admissible; the space $F^{*}$ also becomes a Banach algebra under the product

$$
(\mu \nu)(f)=\mu\left(f_{\nu}\right) \quad \text { for all } \quad f \in F .
$$

For more details, the reader is directed to [2, pages 72-78]. As we have already seen, $L U C(G)$ is admissible. Other examples are the space $W A P(G)$ of weakly almost periodic functions on $G$, and the space $A P(G)$ of almost periodic functions on $G$. These spaces are

$$
\begin{aligned}
W A P(G) & =\left\{f \in C(G): f_{G} \text { is weakly relatively compact }\right\}, \\
A P(G) & =\left\{f \in C(G): f_{G} \text { is norm relatively compact }\right\}, \text { where } \\
f_{G} & =\left\{f_{s}: s \in G\right\} .
\end{aligned}
$$

Let $\mathcal{A}$ denote each of the Banach algebras $L^{1}(G), M(G), L^{1}(G)^{* *}$, and $F^{*}$, where $F$ is an admissible subspace of $C(G)$ with $A P(G) F \subseteq F$. Apart from $L^{1}(G)$, the algebra $\mathcal{A}$ is the dual of some Banach space of functions of $G$, which we shall denote by $\mathcal{F}$. In the case of $L^{1}(G)$, we let $\mathcal{F}=C_{0}(G)$. When $\mathcal{A}=F^{*}$ or $M(G)$, the group $G$ may be embedded continuously into $\mathcal{A}$ by the mapping $e: G \rightarrow \mathcal{A}$ which is defined by

$$
e(s)(f)=f(s) \quad \text { for all } \quad s \in G \text { and } f \in \mathcal{F} .
$$

We recall that an element $\mu$ of $\mathcal{A}$ is left invariant if

$$
\mu\left(f_{s}\right)=\mu(f) \text { for all } f \in \mathcal{F} \text { and } s \in G .
$$

When $L^{1}(G) * \mathcal{F} \subseteq \mathcal{F}$, we say that $\mu \in \mathcal{A}$ is topologically left invariant if

$$
\mu(\phi * f)=\left(\int_{G} \phi(s) d \lambda(s)\right) \mu(f) \text { for all } \phi \in L^{1}(G) \text { and } f \in \mathcal{F} .
$$

The notions of left invariance and topological left invariance are equivalent when $\mathcal{F} \subseteq L U C(G)$. But this is not so if $\mathcal{F}=L^{\infty}(G)$. We say that $\mathcal{F}$ is amenable if there is a non-zero left invariant (or equivalently a topologically left invariant) element in $\mathcal{A}$. When $G$ is a compact topological group, $C_{0}(G)=C(G)$ is amenable since $\lambda \in C(G)^{*}$. The spaces $W A P(G)$ and $A P(G)$ are always amenable. But this is not so for $L U C(G)$ and $L^{\infty}(G)$; for example, when $G$ is the free group on two generators (see [2, Example 3.4(e)]). So we say that the group $G$ is amenable if $L^{\infty}(G)$, or equivalently $L U C(G)$, is amenable. See [2] or [7].

We shall also need representations of $G$ on $\mathbb{C}^{n}$. Recall that a representation of $G$ on a Hilbert space $H$ is a homomorphism of $G$ into the semigroup of bounded operators on $H$. We say that $U$ is continuous when the function $s \mapsto U(s) \bar{x}$ is continuous on $G$ for each $\bar{x} \in H$. We say that $U$ is irreducible when $\{0\}$ and $H$ are the only invariant (closed) subspaces under all $U(s)$, i.e., there is no (closed) 
subspace $E$ other than $\{0\}$ and $H$ satisfying $U(s) E \subseteq E$ for all $s \in G$. We also recall that there is a one-to-one correspondence between the representations of $L^{1}(G)$ on $H$ and those of $G$ on $H$. This is given by the formula

$$
\langle U(\phi) \bar{x}, \bar{y}\rangle=\int_{G}\langle U(s) \bar{x}, \bar{y}\rangle \phi(s) d \lambda(s) \quad \phi \in L^{1}(G), \bar{x}, \bar{y} \in H ;
$$

see [8, Section 22]. Since we shall be concerned solely with representations $U$ on $H=\mathbb{C}^{n}$, we fix a basis $\left\{\bar{x}_{1}, \bar{x}_{2}, \ldots, \bar{x}_{n}\right\}$ for $\mathbb{C}^{n}$, and correspond to $U$ the matrix representation $U=\left(u_{i j}\right)_{i, j=1}^{n}$, where $u_{i j}$ are the coordinate functions defined on $G$ by

$$
u_{i j}(s)=\left\langle U(s) \bar{x}_{i}, \bar{y}_{j}\right\rangle .
$$

Note that the letter $U$ is used to denote the representations of $G, L^{1}(G)$, and their corresponding matrices, but we promise the reader that this will cause no confusion.

\section{LEFT IDEALS OF FINITE-DIMENSION}

The notion of $\chi$-invariance, where $\chi$ is a continuous character of $G$ was introduced in [3] to determine the minimal left ideals of $\mathcal{A}$ when $G$ is abelian. In this section, we generalize this notion by defining the $U$-invariant vectors of $\mathcal{A}^{n}$, where $U$ is a representation of $G$ on $\mathbb{C}^{n}$. The $U$-invariance is essential to determine the finitedimensional left ideals of these algebras. Some of the arguments in Theorems 1 and 2 have already been in [1]; they are, however, simplified here with the help of the $U$-invariance. Before we state our main definition, we introduce the following notations.

Notations. Let $\mu \in \mathcal{A}, f \in \mathcal{F}, \bar{\mu}=\left(\mu_{i}\right)_{i=1}^{n}$ be a column vector in $\mathcal{A}^{n}$, and $A$ be an $n \times n$ matrix with entries $a_{i j}(i, j=1, \ldots, n)$ in $\mathcal{F}$. Then we write $\mu(A)=$ $\left(\mu\left(a_{i j}\right)\right)_{i, j=1}^{n}, \bar{\mu}(f)$ is the column vector $\left(\mu_{i}(f)\right)_{i=1}^{n}$ of $\mathbb{C}^{n},(A) \bar{\mu}$ is the column vector $\left(\sum_{j=1}^{n} \mu_{j}\left(a_{i j}\right)\right)_{i=1}^{n}$ of $\mathbb{C}^{n}$ (note that this is obtained with the matrix multiplication relative to the product given by the duality between $\mathcal{A}$ and $\mathcal{F}$ ), and $\mu \bar{\mu}$ is the column vector $\left(\mu \mu_{i}\right)_{i=1}^{n}$ of $\mathcal{A}^{n}$. Furthermore, when $A$ and $B$ are $n \times n$ matrices whose entries are measurable functions on $G$ then, whenever the integrals exist, we write

$$
\begin{aligned}
A \cdot B & =\int_{G} A(t) B(t) d \lambda(t), \\
A * B(s) & =\int_{G} A(t) B\left(t^{-1} s\right) d \lambda(t), \\
A \tilde{\star} B(s) & =\int_{G} B\left(t^{-1} s\right) A(t) d \lambda(t) .
\end{aligned}
$$

Definition. We say that a vector $\bar{\mu}$ of $\mathcal{A}^{n}$ is $U$-invariant if there exists a continuous and bounded representation $U$ of $G$ on $\mathbb{C}^{n}$ such that

$$
\bar{\mu}\left(f_{s}\right)=U\left(s^{-1}\right) \bar{\mu}(f) \quad \text { for each } \quad f \in \mathcal{F} \text { and } s \in G .
$$

When $\mathcal{A}=L^{1}(G)^{* *}$, we say that $\bar{\mu}$ be topologically $U$-invariant if

$$
\bar{\mu}(\phi * f)=U(\phi) \bar{\mu}(f) \quad \text { for each } \quad f \in L^{\infty}(G) \text { and } \phi \in L^{1}(G) .
$$

Let $\widetilde{U}$ be defined on $G$ by $\widetilde{U}(s)=U\left(s^{-1}\right)$. A direct computation leads to the following lemma. We omit the proof. 
Lemma 1. Let $A$ be an $n \times n$ matrix with entries in $\mathcal{F}$, and for $s \in G$, let $A_{s}$ be the matrix whose entries are the left translates of those of $A$ by s. Let $\Phi$ be an $n \times n$ matrix with entries in $L^{1}(G)$.

(1) If $\bar{\mu} \in \mathcal{A}^{n}$ is $U$-invariant, then $\left(A_{s}\right) \bar{\mu}=(A \widetilde{U}(s)) \bar{\mu}$.

(2) If $\mathcal{A}=L^{1}(G)^{* *}$ and $\bar{\mu} \in \mathcal{A}^{n}$ is topologically $U$-invariant, then $(\Phi * A) \bar{\mu}=(\Phi \cdot A() U.) \bar{\mu}$ and $(\Phi \tilde{*} A) \bar{\mu}=(A(\Phi \cdot U)) \bar{\mu}$, where $\Phi \cdot A()$.$U is$ the matrix-valued function defined on $G$ by $\Phi \cdot A(s) U=\int_{G} \Phi(t) A(s) U(t) d \lambda(t)$.

Lemma 2. Let $U$ be a continuous and bounded representation of $G$ on $\mathbb{C}^{n}$, and let $I$ be the identity representation. Let $\bar{\mu}$ and $\bar{\nu}$ be in $\mathcal{A}^{n}$ such that $\bar{\mu}(f)=(f U) \bar{\nu}$ for all $f \in \mathcal{F}$; or equivalently, $\bar{\nu}(f)=(f \widetilde{U}) \bar{\mu}$ for all $f \in \mathcal{F}$. Then

(1) $\bar{\nu}$ is $I$-invariant if and only if $\bar{\mu}$ is $U$-invariant,

(2) when $\mathcal{A}=L^{1}(G)^{* *}, \bar{\nu}$ is topologically I-invariant if and only if $\bar{\mu}$ is topologically $U$-invariant.

Remark. Observe that $(f U) \bar{\nu}$ and $(f \widetilde{U}) \bar{\mu}$ are well defined in the lemma. This is due to the fact that the coordinate functions of $U$ and $\widetilde{U}$ are almost periodic (which is easy to verify, or see [1, Lemma 1]) and the extra assumption that $A P(G) F \subseteq F$.

Proof of Lemma 2. Let $\bar{\nu} \in \mathcal{A}^{n}$ be $I$-invariant, $s \in G$ and $f \in \mathcal{F}$. Then, by Lemma 1 ,

$$
\bar{\mu}\left(f_{s}\right)=\left(f_{s} U\right) \bar{\nu}=\left(\widetilde{U}(s) f_{s} U_{s}\right) \bar{\nu}=\widetilde{U}(s)(f U) \bar{\nu}=\widetilde{U}(s) \bar{\mu}(f) .
$$

So $\bar{\mu}$ is $U$-invariant. For the converse, let $\bar{\mu}$ be $U$-invariant, $s \in G$ and $f \in \mathcal{F}$. Then

$$
\bar{\nu}\left(f_{s}\right)=\left(f_{s} \widetilde{U}\right) \bar{\mu}=\left(f_{s} \widetilde{U}_{s} U(s)\right) \bar{\mu}=(f \widetilde{U} U(s) \widetilde{U}(s)) \bar{\mu}=(f \widetilde{U}) \bar{\mu}=\bar{\nu}(f) .
$$

So $\bar{\nu}$ is $I$-invariant.

Statement (2) follows with the help of statement (2) of Lemma 1 . Let $\bar{\nu}$ be topologically $I$-invariant, $\phi \in L^{1}(G)$ and $f \in L^{\infty}(G)$. We remark first that

$$
\begin{aligned}
(\phi * f) U(s) & =U(s) \int_{G} \phi(t) f\left(t^{-1} s\right) d \lambda(t) \\
& =\int_{G} U(t) U\left(t^{-1} s\right) \phi(t) f\left(t^{-1} s\right) d \lambda(t)=(\phi U) *(f U)(s) .
\end{aligned}
$$

Therefore,

$$
\begin{aligned}
\bar{\mu}(\phi * f) & =((\phi * f) U) \bar{\nu}=((\phi U) *(f U)) \bar{\nu}=((\phi U) \cdot(f U)(.) I) \bar{\nu} \\
& =\left(\int_{G} U(s) \phi(s) d \lambda(s)\right)(f U) \bar{\nu}=U(\phi) \bar{\mu}(f),
\end{aligned}
$$

and so $\bar{\mu}$ is topologically $U$-invariant.

For the converse, let $\bar{\mu}$ be topologically $U$-invariant, $f \in L^{\infty}(G)$ and $\phi \in L^{1}(G)$. Then, for each $s \in G$,

$$
\begin{aligned}
(\phi * f) \widetilde{U}(s) & =\widetilde{U}(s) \int_{G} \phi(t) f\left(t^{-1} s\right) d \lambda(t) \\
& =\int_{G} \widetilde{U}\left(t^{-1} s\right) \widetilde{U}(t) \phi(t) f\left(t^{-1} s\right) d \lambda(t)=(\phi \widetilde{U}) \tilde{*}(f \widetilde{U})(s),
\end{aligned}
$$


and so

$$
\begin{aligned}
\bar{\nu}(\phi * f) & =((\phi * f) \widetilde{U}) \bar{\mu}=((\phi \widetilde{U}) \tilde{*}(f \widetilde{U})) \bar{\mu}=((f \widetilde{U})((\phi \widetilde{U}) \cdot U)) \bar{\mu} \\
& =\left((f \widetilde{U}) \int_{G} \phi(s) \widetilde{U}(s) U(s) d \lambda(s)\right) \bar{\mu} \\
& =\left(\int_{G} \phi(s) d \lambda(s)\right)(f \widetilde{U}) \bar{\mu}=\left(\int_{G} \phi(s) d \lambda(s)\right) \bar{\nu}(f) .
\end{aligned}
$$

So $\bar{\nu}$ is topologically $I$-invariant.

Theorem 1. Let $G$ be a locally compact group. Let $\bar{\mu} \in \mathcal{A}^{n}$ and $M$ be the linear span of the coordinates $\mu_{1}, \mu_{2}, \ldots, \mu_{n}$ of $\bar{\mu}$. Let $U$ be a continuous and bounded representation of $G$ on $\mathbb{C}^{n}$. Then $M$ is a left ideal of $\mathcal{A}$ of dimension less or equal to $n$ in each of the following cases:

(1) $F$ is amenable, $\mathcal{A}=F^{*}$ and $\bar{\mu}$ is $U$-invariant.

(2) $G$ is amenable, $\mathcal{A}=L^{1}(G)^{* *}$ and $\bar{\mu}$ is topologically $U$-invariant.

(3) $G$ is compact, $\mathcal{A}=L^{1}(G)$ or $M(G)$, and $\bar{\mu}$ is $U$-invariant.

Furthermore, $M$ is minimal and of dimension $n$ when $U$ is irreducible.

Proof. We consider only the first two statements. The proof of statement (3) is similar. Let $\mathcal{A}=F^{*}$, and let $\bar{\mu}$ be $U$-invariant. That $M$ is a left ideal follows directly from the lemma above. Let $\mu$ be arbitrary in $F^{*}$ and $f \in F$. Then $(\mu \bar{\mu})(f)=\mu\left(f_{\bar{\mu}}\right)$, where $f_{\bar{\mu}}(s)=\bar{\mu}\left(f_{s}\right)=\widetilde{U}(s) \bar{\mu}(f)$, and so

$$
\mu \bar{\mu}(f)=\mu(\widetilde{U} \bar{\mu}(f))=\mu(\widetilde{U}) \bar{\mu}(f) .
$$

Thus, $\mu \bar{\mu}=\mu(\widetilde{U}) \bar{\mu}$, which means obviously that $M$ is a left ideal of $F^{*}$.

For statement $(2)$, let $\mu \in L^{1}(G)^{* *}$ and $f \in L^{\infty}(G)$. Then $(\mu \bar{\mu})(f)=\mu\left(f_{\bar{\mu}}\right)$, where

$$
\begin{aligned}
& f_{\bar{\mu}}(\phi)=\bar{\mu}(\hat{\phi} * f)=U(\hat{\phi}) \bar{\mu}(f), \text { and } \\
& U(\hat{\phi})=\int_{G} U(s) \hat{\phi}(s) d \lambda(s)=\int_{G} \widetilde{U}(s) \phi(s) d \lambda(s)=\widetilde{U}(\phi) \text { for all } \phi \in L^{1}(G) .
\end{aligned}
$$

Thus, $(\mu \bar{\mu})(f)=\mu\left(f_{\bar{\mu}}\right)=\mu(\widetilde{U}) \bar{\mu}$, and so $M$ is a left ideal of $L^{1}(G)^{* *}$. That $M$ is of dimension less or equal to $n$ is clear in each case.

Suppose now that $U$ is irreducible, and let us prove that $M$ is minimal. We start with the algebra $F^{*}$. Let $\mu \in M$ be arbitrary, and write $\mu=\sum_{i=1}^{n} x_{i} \mu_{i}=\bar{\mu} \underline{x}=\bar{x} \underline{\mu}$, where $\underline{x}=\left(x_{1}, x_{2}, \ldots, x_{n}\right)$ is a non-zero vector of $\mathbb{C}^{n}$. For each vector $\bar{y} \in \mathbb{C}^{n}$, we can find $s_{1}, s_{2}, \ldots, s_{k}$ in $G$ and $\alpha_{1}, \alpha_{2}, \ldots, \alpha_{k}$ in $\mathbb{C}$ such that

$$
\sum_{i=1}^{n} \alpha_{i} \widetilde{U}\left(s_{i}\right) \bar{x}=\bar{y}
$$

since $\widetilde{U}$ is also irreducible. It follows that

$$
\begin{aligned}
\sum_{i=1}^{n} \alpha_{i} e\left(s_{i}\right) \mu & =\sum_{i=1}^{n} \alpha_{i} e\left(s_{i}\right)(\bar{\mu} \underline{x})=\sum_{i=1}^{n} \alpha_{i}\left(e\left(s_{i}\right) \bar{\mu}\right) \underline{x} \\
& =\sum_{i=1}^{n} \alpha_{i}\left(\widetilde{U}\left(s_{i}\right) \bar{\mu}\right) \underline{x}=\sum_{i=1}^{n} \alpha_{i}\left(\widetilde{U}\left(s_{i}\right) \bar{x}\right) \underline{\mu}=\bar{y} \underline{\mu}
\end{aligned}
$$


(remember that $e(s) \in F^{*}$ and $e(s)(f)=f(s)$ for $s \in G$ and $f \in F$ ). This means first that $F^{*} \mu=M$, and so $M$ is minimal. Secondly, if $\bar{y} \in \mathbb{C}^{n}$ is such that $\bar{y} \underline{\mu} \neq \overline{0}$, then this argument shows also that

$$
\sum_{i=1}^{n} \alpha_{i} e\left(s_{i}\right) \bar{\mu} \underline{x}=\bar{y} \underline{\mu} \neq 0,
$$

and implies that $\bar{\mu} \underline{x} \neq 0$. So the elements $\mu_{1}, \mu_{2}, \ldots, \mu_{n}$ are linearly independent and $M$ is of dimension $n$.

In $L^{1}(G)^{* *}$, the corresponding representation $U$ of $L^{1}(G)$ is also irreducible, and so we can find, for each $\bar{y} \in \mathbb{C}^{n}, \phi \in L^{1}(G)$ such that $U(\phi) \bar{x}=\bar{y}$. As above, this shows that $M$ is minimal and is of dimension $n$.

Remark. Statement (1) means that $G$ needs to be amenable if $F=L U C(G)$.

Theorem 2. Let $\mathcal{A}$ be $L^{1}(G), M(G)$ or $F^{*}$, and let $M$ be a left ideal of $\mathcal{A}$ of dimension $n$. Then there exist $m$ vectors $\bar{\mu}^{i} \in \mathcal{A}^{n_{i}}$ and $m$ irreducible, unitary, bounded and continuous representations $U^{i}(i=1,2, \ldots, m)$ of $G$ such that

(1) each $\bar{\mu}^{i}$ is $U^{i}$-invariant,

(2) for each $i=1,2, \ldots, m$, the coordinates of $\bar{\mu}^{i}$ span a minimal left ideal $M_{i}$ of $\mathcal{A}$ of dimension $n_{i}$, and

(3) $M=M_{1} \oplus M_{2} \oplus \ldots \oplus M_{m}$.

Proof. In the case of $\mathcal{A}=L^{1}(G)$, we regard $M$ as a left ideal of $M(G)$. This is possible because $M$ is closed and $L^{1}(G)$ is a closed ideal of $M(G)$. We start with a set of elements $\mu_{1}, \mu_{2}, \ldots, \mu_{n}$ which generate $M$, and let $\bar{\mu}=\left(\mu_{i}\right)_{i=1}^{n}$. Then, for each $\mu \in \mathcal{A}$ and for each $i=1,2, \ldots, n$, there exist $a_{i 1}(\mu), a_{i 2}(\mu), \ldots, a_{i n}(\mu) \in \mathbb{C}$ such that

$$
\mu \mu_{i}=a_{i 1}(\mu) \mu_{1}+a_{i 2}(\mu) \mu_{2}+\ldots+a_{i n}(\mu) \mu_{n} .
$$

Put $A(\mu)=\left(a_{i j}(\mu)\right)_{i, j=1}^{n}$. Then, for $\mu$ and $\nu$ in $\mathcal{A}$,

$$
A(\mu \nu) \bar{\mu}=(\mu \nu) \bar{\mu}=\mu(A(\nu) \bar{\mu})=A(\nu)(\mu \bar{\mu})=A(\nu) A(\mu) \bar{\mu} .
$$

Hence, $A(\mu \nu)=A(\nu) A(\mu)$, i.e., $A$ is an antirepresentation of $\mathcal{A}$. Moreover, for each $1 \leq j \leq n$, let $f \in \mathcal{F}$ be such that $\mu_{j}(f)=1$ and $\mu_{k}(f)=0$ for $k \neq j$. Then, for each $1 \leq i \leq n, \mu \mu_{i}(f)=a_{i j}(\mu)$, so

$$
\left|a_{i j}(\mu)\right| \leq\left\|\mu \mu_{i}\right\|\|f\| \leq\|\mu\|\left\|\mu_{i}\right\|\|f\|,
$$

which implies that $A$ is bounded. Since the product in $\mathcal{A}$ is $\sigma(\mathcal{A}, \mathcal{F})$-continuous on the left side, one can also see that the functions $\mu \mapsto a_{i j}(\mu)$ are $\sigma(\mathcal{A}, \mathcal{F})$-continuous. When $\mathcal{A}=L^{1}(G)$, we let $V$ be the antirepresentation of $G$ associated to $A$; and when $\mathcal{F}=F$ is an admissible subspace of $C(G)$, we let

$$
V(s)=\left(a_{i j}(e(s))\right)_{i, j=1}^{n} .
$$

Then $\widetilde{V}$ is a bounded and continuous representation of $G$ in each case. Furthermore, for $f \in \mathcal{F}$, we have

$$
\bar{\mu}\left(f_{s}\right)=e(s) \bar{\mu}(f)=A(e(s)) \bar{\mu}(f)=V(s) \bar{\mu}(f),
$$

i.e., $\bar{\mu}$ is $\widetilde{V}$-invariant. Now it is easy to verify (or see [1, Lemma 3$]$ ) that $\widetilde{V}$ is in fact equivalent to a unitary representation $U$ in the sense that $P \widetilde{V}(s)=U(s) P$ for all $s \in G$, where $P$ is an invertible operator on $\mathbb{C}^{n}$. (This result is also true for the infinite-dimensional representations when $G$ is amenable; see [9] or [7].) Put 
$\bar{\gamma}=P \bar{\mu}$. It is clear that the coordinates of $\bar{\gamma}$ also generate the ideal $M$. We have also

$$
\bar{\gamma}\left(f_{s}\right)=P \bar{\mu}\left(f_{s}\right)=P V(s) \bar{\mu}(f)=\widetilde{U}(s) P \bar{\mu}(f)=\widetilde{U}(s) \bar{\gamma}(f),
$$

and so $\bar{\gamma}$ is $U$-invariant. Since $U$ is unitary, it follows by $[8,21.40(\mathrm{a})]$ that $U$ is a direct sum of continuous, irreducible representations $U^{1}, U^{2}, \ldots$, and $U^{m}$. This means that $\mathbb{C}^{n}$ is the direct sum of some invariant subspaces $H_{i}$, and each $U^{i}$ is the restriction of $U$ to $H_{i}(i=1,2, \ldots, m)$. For each $f \in \mathcal{F}$, we write $\bar{\gamma}(f)=\sum_{i} \bar{\mu}^{i}(f)$. Then

$$
\sum_{i=1}^{m} \bar{\mu}^{i}\left(f_{s}\right)=\bar{\gamma}\left(f_{s}\right)=\widetilde{U}(s) \bar{\gamma}(f)=\sum_{i=1}^{m} \widetilde{U}^{i}(s) \bar{\mu}^{i}(f) .
$$

It follows that, for each $i=1,2, \ldots, m, \bar{\mu}^{i}\left(f_{s}\right)=\widetilde{U}^{i}(s) \bar{\mu}^{i}(f)$. This yields statement (1). Statement (2) follows from Theorem 1. Statement (3) is clear.

Remark. In $L^{1}(G)^{* *}$, the situation is slightly different. In fact, one can also produce finite-dimensional left ideals with the use of the right annihilators of $L^{1}(G)^{* *}$. These are elements $\mu$ in $L^{1}(G)^{* *}$ which satisfy $L^{1}(G)^{* *} \mu=\{0\}$; see [6]. In such a situation, the representation $\widetilde{V}$ is trivial in the proof above.

Theorem 3. Let $M$ be a left ideal of $L^{1}(G)^{* *}$ of dimension $n$, and suppose that $M$ contains l linearly independent right annihilators $\gamma_{1}, \gamma_{2}, \ldots, \gamma_{l}$ of $L^{1}(G)^{* *}$. Then there exist $m$ vectors $\bar{\mu}^{i} \in\left(L^{1}(G)^{* *}\right)^{n_{i}}$ and $m$ irreducible, unitary, bounded and continuous representations $U^{i}(i=1,2, \ldots, m)$ of $G$ such that

(1) each $\bar{\mu}^{i}$ is topologically $U^{i}$-invariant,

(2) for each $i=1,2, \ldots, m$, the coordinates of $\bar{\mu}^{i}$ span a minimal left ideal $M_{i}$ of $L^{1}(G)^{* *}$ of dimension $n_{i}$, and

(3) $M=\mathbb{C} \gamma_{1} \oplus \mathbb{C} \gamma_{2} \oplus \ldots \oplus \mathbb{C} \gamma_{l} \oplus M_{1} \oplus M_{2} \oplus \ldots \oplus M_{m}$.

Proof. We take $n-l$ linearly independent elements $\mu_{1}, \mu_{2}, \ldots, \mu_{n-l}$ in $M$ which are not right annihilators of $L^{1}(G)^{* *}$, let $\bar{\mu}=\left(\mu_{i}\right)_{i=1}^{n-l}$. Then, form the matrices $A(\mu)$ such that $\mu \bar{\mu}=A(\mu) \bar{\mu}$ for $\mu \in L^{1}(G)^{* *}$, restrict $A$ to $L^{1}(G)$, and let $\widetilde{V}$ be the corresponding representation of $G$. Then, for $\phi \in L^{1}(G)$ and $f \in L^{\infty}(G)$, we have

$$
\bar{\mu}(\phi * f)=\bar{\mu}(\hat{\hat{\phi}} * f)=\hat{\phi} \bar{\mu}(f)=A(\hat{\phi}) \bar{\mu}(f)=V(\hat{\phi}) \bar{\mu}(f)=\widetilde{V}(\phi) \bar{\mu}(f),
$$

and so $\bar{\mu}$ is topologically $\widetilde{V}$-invariant. The proof is completed as that of Theorem 2.

Corollary. Let $G$ be a locally compact group. Then

(1) finite-dimensional (left) ideals exist in $M(G)$ and $L^{1}(G)$ if and only if $G$ is compact,

(2) finite-dimensional left ideals exist in $L U C(G)^{*}$ if and only if $G$ is amenable,

(3) finite-dimensional left ideals which are not generated by right annihilators of $L^{1}(G)^{* *}$ exist in $L^{1}(G)^{* *}$ if and only if $G$ is amenable.

Proof. This follows from Lemma 2 and Theorems 2 and 3.

Remark. The finite-dimensional right ideals in $W A P(G)^{*}$ are determined in the same way because the two Arens product conicide in this case; see [2, Section 4.2]. When $G$ is compact, one proceeds also in the same way to find these ideals in $L^{1}(G)$ and $M(G)$. These facts were already observed for the minimal right ideals in [1, 
Section 4]. However, in [4, Remark 2.7(b)], we have proved that the non-trivial right ideals are all of infinite dimension in $L U C(\mathbb{Z})^{*}=\ell^{\infty}(\mathbb{Z})^{*}$, where $\mathbb{Z}$ is the additive group of the integers. In [1, Section 4], we have given a class of locally compact abelian groups, which includes $\mathbb{Z}$, for which the non-trivial right ideals are all of infinite dimension in $L U C(G)^{*}$. Now we can prove that, for a locally compact abelian group $G$, the finite-dimensional right ideals exist in $L U C(G)^{*}$ if and only if $G$ is compact. We hope to publish this result in another paper.

The author wishes to thank the referee for the very careful reading the paper was given.

\section{REFERENCES}

[1] J. W. Baker and M. Filali, Minimal ideals in group algebras and their biduals, Math. Proc. Cambridge Philos. Soc. 120 (1996), 475-488. CMP 96:12

[2] J. F. Berglund, H. D. Junghenn and P. Milnes, Analysis on Semigroups: Function Spaces, Compactifications, Representations, Wiley, New York, 1989. MR 91b:43001

[3] M. Filali, The ideal structure of some Banach algebras, Math. Proc. Cambridge Philos. Soc. 111 (1992), 567-576. MR 93e:22005

[4] M. Filali, Linear equations in $B(\mathbb{Z})^{*}$, Proc. Roy. Soc. Edinburgh Sect. A 123A (1993), 10011009. MR 95j:39035

[5] M. Filali, Minimal left ideals in the group algebra and its second conjugate, Mathematica Japonica (to appear).

[6] E. E. Granirer, The radical of $L^{\infty}(G)^{*}$, Proc. Amer. Math. Soc. 41 (1973), 321-324. MR 48:4646

[7] F. P. Greenleaf, Invariant means on topological groups and their applications, Van Nostrand, New York, 1969. MR 40:4776

[8] E. Hewitt and K. A. Ross, Abstract harmonic analysis I, Springer-Verlag, Berlin,, 1963, 1970. MR 28: 158

[9] B. Sz.-Nagy, Uniformly bounded linear transformations in Hilbert space, Acta Math. (Szeged) 11 (1947), 152-157.

Department of Mathematical Sciences, University of Oulu, SF 90570 Finland

E-mail address: mfilali@cc.oulu.fi 